\title{
Differential Cryptanalysis of T-Function Based Stream Cipher TSC-4
}

\author{
Haina Zhang ${ }^{1}$ and Xiaoyun Wang ${ }^{2, \star}$ \\ ${ }^{1}$ Key Laboratory of Cryptologic Technology and Information Security, \\ Ministry of Education, Shandong University, Jinan 250100, P.R. China \\ hnzhang@math.sdu .edu.cn \\ 2 Tsinghua University, Beijing 100087, P.R. China \\ xiaoyunwang@tsinghua.edu.cn
}

\begin{abstract}
TSC-4 is a T-function based stream cipher with 80-bit key, and proposed as a candidate for ECRYPT eStream project. In this paper, we introduce a differential method to analyze TSC-4. Our attack is based on the vulnerable differential characteristics in the state initialization of TSC-4, and for the chosen IV pairs, the differential probability is up to $2^{-15.40}$ in the case of weak keys. We show that there are about $2^{72}$ weak keys among the total $2^{80}$ keys. To recover 8 bits of a weak key needs about $2^{40.53}$ chosen IV pairs. After that, we can search the other 72 key bits by an exhaustive attack.
\end{abstract}

Keywords: Differential cryptanalysis, T-function, stream cipher, chosen IV attack, TSC-4.

\section{Introduction}

For a long time, a classical approach for designing stream ciphers is to utilize the Linear Feedback Shift Registers (LFSR) combined with nonlinear Boolean functions. Recently, a new primitive has been introduced to replace the LFSR, which is the T-function (Triangular-function) proposed by Klimov and Shamir [45|6]. T-function is a nonlinear function which has some nice properties, such as the operations both available on processors and software implementations, and one single cycle by the appropriate choice, etc. There are some interesting researches on this area in recent years.

At FSE 2005, Hong et al. proposed a new class of single T-functions with S-box application [1], and described two stream ciphers TSC-1 and TSC-2. At Asiacrypt 2004, Mitra and Sarkar described a time-memory trade-off attack 8 to break some stream ciphers proposed by Klimov and Shamir. Recently, Kunzli, Junod and Meier proposed the distinguishing attacks [7] applicable to several Tfunction based stream ciphers (TSC). Based on the above researches, Hong et al. proposed a new TSC version called TSC-3 [2], as a candidate to eStream project.

\footnotetext{
* Supported by the National Natural Science Foundation of China (NSFC Grant No.90604036) and 973 Project (No.2007CB807902).
} 
In 2005 and 2006, Muller and Peyrin 1011 introduced a linear cryptanalysis attack on the TSC family TSC-1, TSC-2, TSC-3 together with Klimov and Shamir's ciphers. This linear correlation attack can recover the full secret keys of these stream ciphers. Soon, Dukjae Moon et al. twisted TSC-3 and proposed the latest version TSC-4 [9] to prevent the distinguishing attack, the time-memory trade-off attack and the linear correlation attack described in above literatures.

At Indocrypt 2006, Fischer, Meier and Berbain et al. 3 presented a nonrandomness behavior of the full eight-round state initialization for TSC-4. The non-randomness can be detected in the initial state with about 1000 inputs, however, no bias in the keystream of TSC-4 resulting from this non-randomness has been detected yet. So, no substantial attack has been found from the keystream until now.

In this paper, we present an efficient chosen IV differential attack on TSC-4 with weak keys based on two special differential characteristics in the state initialization. The outline of our attack is as follows. Firstly, construct two special differential characteristics in the state initialization of TSC-4. Secondly, investigate what keys and IVs can result in the high occurrence probability of two differential characteristics, and find out the serious non-randomness behavior of the initial state under these weak keys and chosen IVs. Finally, utilizing $2^{40.53}$ chosen IV pairs, we can identify a weak key and recover 8 bits of the key, and recover the full 80-bit weak key by searching exhaustively the other 72 key bits.

This paper is organized as follows. In Section 2, a brief description of TSC-4 is given. Section 3 introduces two special differential characteristics according to the structure of TSC-4. Section 4 presents the chosen IV differential attack on TSC-4. Finally, we conclude the paper in Section 5.

\section{A Brief Description of TSC-4}

TSC-4 is a synchronous stream cipher optimized for constrained hardware, including the cipher body and the state initialization. The structure of TSC-4 is illustrated in Fig. 1. Its internal state consists of two states $X$ and $Y$ of $4 \times 32$ bits each, denoted $X=\left(x_{3}, x_{2}, x_{1}, x_{0}\right)^{T}$ and $Y=\left(y_{3}, y_{2}, y_{1}, y_{0}\right)^{T}$, where $x_{k}$ and $y_{k}(k=0, \cdots, 3)$ are 32 -bit words. Let $[x]_{i}$ denote the $i$-th least significant bit of a 32-bit word $x$, then the $i$-th bit-slice of state $X$ and the $i$-th bit-slice of $Y$ are defined as

$$
[X]_{i}=\sum_{k=0}^{3}\left[x_{k}\right]_{i} 2^{k}, \quad[Y]_{i}=\sum_{k=0}^{3}\left[y_{k}\right]_{i} 2^{k}, \quad i=0, \cdots 31 .
$$

\subsection{Cipher Body}

We first describe two functions of $X$ and $Y$. In the case of state $X$, a 32-bit parameter $\alpha_{1}(X)$ is computed as a function of $X$. It is defined by $\alpha_{1}(X)=$ $(p+c) \oplus p \oplus 2 s$ with $p=x_{0} \wedge x_{1} \wedge x_{2} \wedge x_{3}$ and $s=x_{0}+x_{1}+x_{2}+x_{3}$ and constant $c_{X}=0 \times 51291089$. Here $\wedge, \oplus$ and + denote bitwise AND, bitwise XOR 


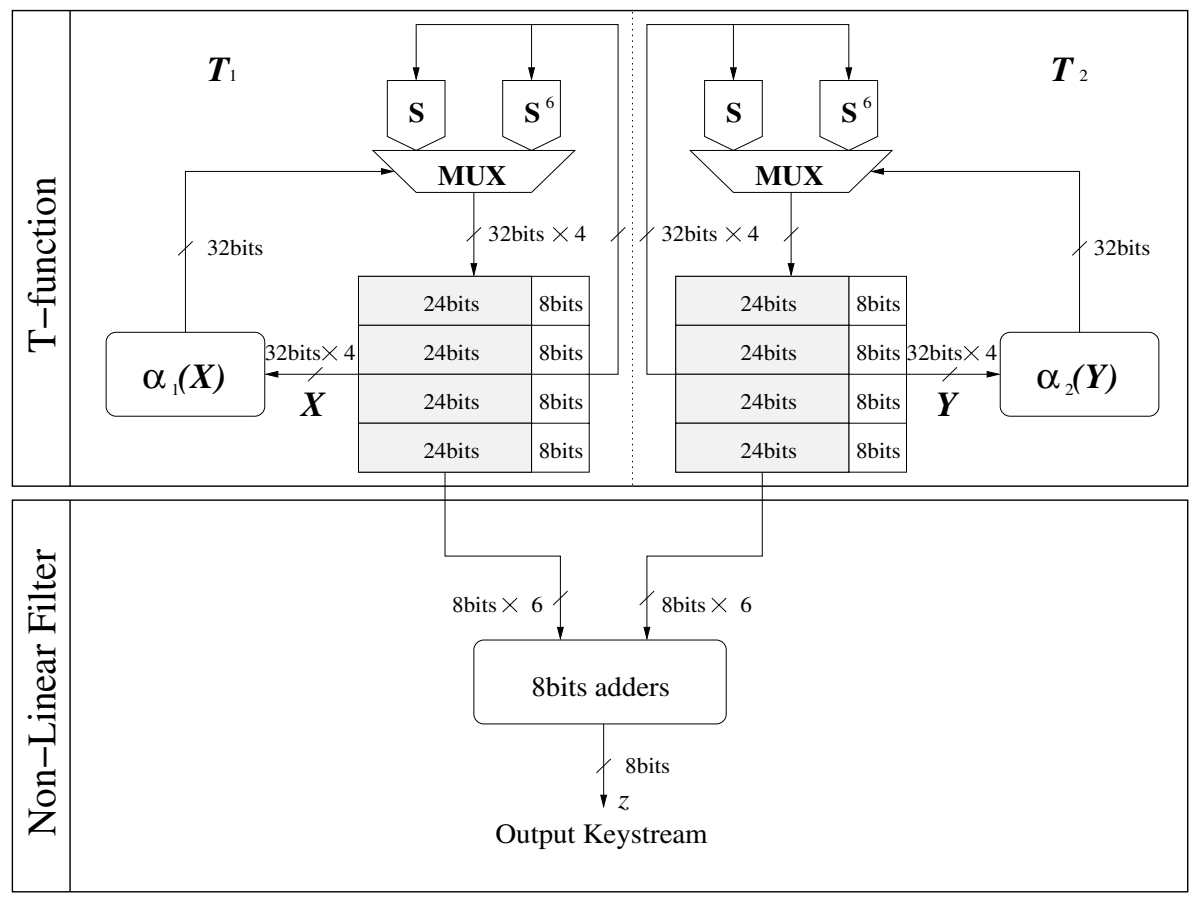

Fig. 1. The structure of TSC-4

and addition operation modulo $2^{32}$ respectively. The 32-bit parameter $\alpha_{2}(Y)$ is defined similarly to $\alpha_{1}(X)$ except for the constant $c_{Y}=0 \mathrm{x} 12910895$.

In the cipher body, bit-slices $[X]_{i}$ and $[Y]_{i}$ are always mapped by two singlecycle $S$-boxes. The $S$-boxes are defined as

$$
\begin{gathered}
S[16]=\{9,2,11,15,3,0,14,4,10,13,12,5,6,8,7,1\}, \\
S^{6}[16]=\{6,13,8,0,5,12,1,11,4,14,3,10,15,7,2,9\} .
\end{gathered}
$$

Now T-functions $T_{1}$ and $T_{2}$ on input states $X$ and $Y$ are defined as

$$
\begin{aligned}
& {\left[T_{1}(X)\right]_{i}=\left\{\begin{array}{lll}
S\left([X]_{i}\right) & \text { if } & {\left[\alpha_{1}(X)\right]_{i}=1,} \\
S^{6}\left([X]_{i}\right) & \text { if } & {\left[\alpha_{1}(X)\right]_{i}=0 .}
\end{array}\right.} \\
& {\left[T_{2}(Y)\right]_{i}=\left\{\begin{array}{lll}
S\left([Y]_{i}\right) & \text { if } & {\left[\alpha_{2}(Y)\right]_{i}=1,} \\
S^{6}\left([Y]_{i}\right) & \text { if } & {\left[\alpha_{2}(Y)\right]_{i}=0 .}
\end{array}\right.}
\end{aligned}
$$

Where the bit-slices $\left[T_{1}(X)\right]_{i}$ and $\left[T_{2}(Y)\right]_{i}(i=0,1, \cdots, 31)$ are regarded as 4-bit integers similar to equations $(1)$. Then $[X]_{i}$ will be replaced by $\left[T_{1}(X)\right]_{i}$, and $[Y]_{i}$ will be replaced by $\left[T_{2}(Y)\right]_{i}$ respectively, in this way, the states $X$ and $Y$ are updated by T-function $T_{1}$ and $T_{2}$.

The non-linear filter in Fig. 1. produces the actual output keystream from the current internal states $X$ and $Y$. We divide 32-bit word $x_{k}$ into four bytes 
$x_{k, 0}, x_{k, 1}, x_{k, 2}, x_{k, 3}$, where $x_{k, 0}$ is the least significant byte, and $x_{k, 3}$ is the most significant byte. This is similar to $y_{k, l}(0 \leq k, l \leq 3)$. Then we compute six 8-bit temporary variables $a_{0}, \cdots, a_{5}$ as follows:

$$
\begin{aligned}
& a_{0}=x_{3,3}+y_{1,1}, \\
& a_{1}=x_{0,3}+y_{2,1}, \\
& a_{2}=x_{2,2}+y_{3,2}, \\
& a_{3}=x_{1,2}+y_{0,2}, \\
& a_{4}=x_{3,1}+y_{2,3}, \\
& a_{5}=x_{0,1}+y_{1,3},
\end{aligned}
$$

where, + denotes the addition operation modulo $2^{8}$. Now the 8-bit keystream $z$ is defined as

$$
z=a_{0} \oplus\left(a_{1}\right)_{\ggg 5} \oplus\left(a_{2}\right)_{\ggg 2} \oplus\left(a_{3}\right)_{\ggg 5} \oplus\left(a_{4}\right)_{\ggg 6} \oplus\left(a_{5}\right)_{\ggg 2},
$$

where denotes the right rotation.

\subsection{State Initialization}

We now describe how the states $X$ and $Y$ are initialized from a given key and IV.

Key /IV Loading: Let $K=\left(k_{79}, k_{78}, \cdots, k_{1}, k_{0}\right)$ and $I V=\left(i v_{79}, i v_{78}, \cdots, i v_{1}\right.$, $\left.i v_{0}\right)$ be an 80 -bit key and 80 -bit IV respectively. Two 128-bit internal states $X$ and $Y$ are initialized as follows:

$$
\begin{gathered}
X=\left(\begin{array}{l}
x_{3} \\
x_{2} \\
x_{1} \\
x_{0}
\end{array}\right)=\left(\begin{array}{ccccc}
i v_{63} & i v_{62} & \cdots & i v_{33} & i v_{32} \\
i v_{31} & i v_{30} & \cdots & i v_{1} & i v_{0} \\
k_{63} & k_{62} & \cdots & k_{33} & k_{32} \\
k_{31} & k_{30} & \cdots & k_{1} & k_{0}
\end{array}\right), \\
Y=\left(\begin{array}{l}
y_{3} \\
y_{2} \\
y_{1} \\
y_{0}
\end{array}\right)=\left(\begin{array}{cccccc}
k_{47} & k_{46} & \cdots & \cdots & k_{17} & k_{16} \\
k_{15} & \cdots & k_{0} & k_{79} & \cdots & k_{64} \\
i v_{47} & i v_{46} & \cdots & \cdots & i v_{17} & i v_{16} \\
i v_{15} & \cdots & i v_{0} & i v_{79} & \cdots & i v_{64}
\end{array}\right) .
\end{gathered}
$$

Warm-up: Once the internal states are initialized, the $K$ and $I V$ are mixed by the following process,

- Step 1, run cipher body once to produce a single 8-bit output,

- Step 2, rotate $x_{1}$ and $y_{0}$ to the left by 8 bits,

- Step 3, XOR the output $z$ to the least significant 8 bits of $x_{1}$ and $y_{0}$.

The state initialization is completely finished by repeating the above three steps 8 times. 


\section{Two Special Differential Characteristics in the State Initialization}

In this section, we will construct two special differential characteristics which will occur with obvious probability advantage for a large amount of keys.

Suppose that $I V$ and $I V^{\prime}$ are two different IVs, and we denote them as an IV pair $\left(I V, I V^{\prime}\right)$. Let $X=\left(x_{3}, x_{2}, x_{1}, x_{0}\right)^{T}$ and $Y=\left(y_{3}, y_{2}, y_{1}, y_{0}\right)^{T}$ are two 128-bit initial states corresponding to $I V . X^{\prime}=\left(x_{3}^{\prime}, x_{2}^{\prime}, x_{1}^{\prime}, x_{0}^{\prime}\right)^{T}$ and $Y^{\prime}=$ $\left(y_{3}^{\prime}, y_{2}^{\prime}, y_{1}^{\prime}, y_{0}^{\prime}\right)^{T}$ are states related to $I V^{\prime}$. Denote the differential $X \oplus X^{\prime}$ as $\Delta X=\left(\Delta x_{3}, \Delta x_{2}, \Delta x_{1}, \Delta x_{0}\right)^{T}$, and $Y \oplus Y^{\prime}$ as $\Delta Y=\left(\Delta y_{3}, \Delta y_{2}, \Delta y_{1}, \Delta y_{0}\right)^{T}$, where $\Delta x_{k}=x_{k} \oplus x_{k}^{\prime}, \Delta y_{k}=y_{k} \oplus y_{k}^{\prime}, k=0, \cdots, 3$.

All the 16 bytes of $\Delta Y$ can be represented as a matrix $\Delta Y=\left(\Delta y_{k, l}\right)_{4 \times 4}$ with $\Delta y_{k, l}=y_{k, l} \oplus y_{k, l}^{\prime}$, where $0 \leq k, l \leq 3$.

$$
\Delta Y=\left(\begin{array}{c}
\Delta y_{3} \\
\Delta y_{2} \\
\Delta y_{1} \\
\Delta y_{0}
\end{array}\right)=\left(\begin{array}{cccc}
\Delta y_{3,3} & \Delta y_{3,2} & \Delta y_{3,1} & \Delta y_{3,0} \\
\Delta y_{2,3} & \Delta y_{2,2} & \Delta y_{2,1} & \Delta y_{2,0} \\
\Delta y_{1,3} & \Delta y_{1,2} & \Delta y_{1,1} & \Delta y_{1,0} \\
\Delta y_{0,3} & \Delta y_{0,2} & \Delta y_{0,1} & \Delta y_{0,0}
\end{array}\right) .
$$

In the Key/IV loading process, it is obvious that the IV bits $i v_{48}, \cdots, i v_{63}$ (to initial state $X$ ) and $i v_{64}, \cdots, i v_{79}$ (to initial state $Y$ ) occur only one time respectively, and the other IV bits occur twice. This means that one nonzero bit difference in $\left\{i v_{64}, i v_{49}, \cdots, i v_{79}\right\}$ results in only one nonzero difference bit in $\Delta Y$.

We denote the IV differential $I V \oplus I V^{\prime}$ as $\Delta I V=\left(\Delta i v_{79}, \cdots \Delta i v_{0}\right)$ with $\Delta i v_{j}=i v_{j} \oplus i v_{j}^{\prime}$, where $0 \leq j \leq 79$. Then we announce for the same key $K$, the chosen IV pair holds that $I V$ and $I V^{\prime}$ are only different at one bit $\Delta i v_{j_{0}}=1$ and the other 79 bits of them are all the same, where $64 \leq j_{0} \leq 79$. After the key/IV loading process, we can get the initial state of $X$ and $Y$ are $\Delta X^{0}=(0,0,0,0)^{T}$ and $\Delta Y^{0}=\left(0,0,0, \Delta y_{0}^{0}\right)^{T}$ respectively, where $\Delta y_{0}^{0}$ only has one nonzero bit difference (i.e., the hamming weight of $\Delta y_{0}^{0}$ is 1). In this paper, we select $j_{0}=66$ i.e., $\Delta y_{0}^{0}=0 \times 4$. The choice for the position $j_{0}$ depends on the occurrence probability of the expected differential characteristic in the state initialization process, the details are described in Subsection 4.1. The state initialization in Section 2.2 includes 8 warm-up processes, and we denote each warm-up process as one round.

Now we construct two differential characteristics $\Omega_{X}$ and $\Omega_{Y}$, and discuss the interaction between them.

Differential characteristic $\Omega_{X}: \Omega_{X}$ is an 8 -round differential in the state initialization such that the difference of initial state $X$ after Key/IV loading process is $\Delta X^{0}=(0,0,0,0)^{T}$, and the output difference of internal state $X$ after Step 3 at the $i$-th round is $\Delta X^{i}=(0,0,0,0)^{T}, i=1, \cdots, 8$, i.e.,

$$
\Omega_{X}: X^{0} \underset{\text { Round } 1}{\stackrel{\text { Step } 3}{\longrightarrow}} X^{1} \underset{\text { Round } 2}{\stackrel{\text { Step 3 }}{\longrightarrow}} X^{2} \longrightarrow \ldots \frac{\text { Step } 3}{\text { Round } 8} X^{8} .
$$


Differential characteristic $\Omega_{Y}: \Omega_{Y}$ behaves that, the difference of initial state $Y$ after Key/IV loading process is $\Delta Y^{0}=(0,0,0,4)^{T}$, and the output difference of internal state $Y$ after Step 1 before Step 2 at the $i$-th round is $\Delta Y^{i}$ which holds that 6 differential bytes $\Delta Y_{3,2}^{i}, \Delta Y_{2,3}^{i}, \Delta Y_{2,1}^{i}, \Delta Y_{1,3}^{i}, \Delta Y_{1,1}^{i}$ and $\Delta Y_{0,2}^{i}$ are all 0 . Here $i=1, \cdots, 8$.

$$
\Omega_{Y}: Y^{0} \underset{\text { Round } 1}{\stackrel{\text { Step } 1}{\longrightarrow}} \Delta Y^{1} \underset{\text { Round } 2}{\stackrel{\text { Step } 1}{\longrightarrow}} \Delta Y^{2} \longrightarrow \cdots \underset{\text { Round } 8}{\stackrel{\text { Step } 1}{\longrightarrow}} \Delta Y^{8} .
$$

The differential characteristic $\Omega_{Y}$ is illustrated in Fig. 2. The blank means that the difference byte is 0 .

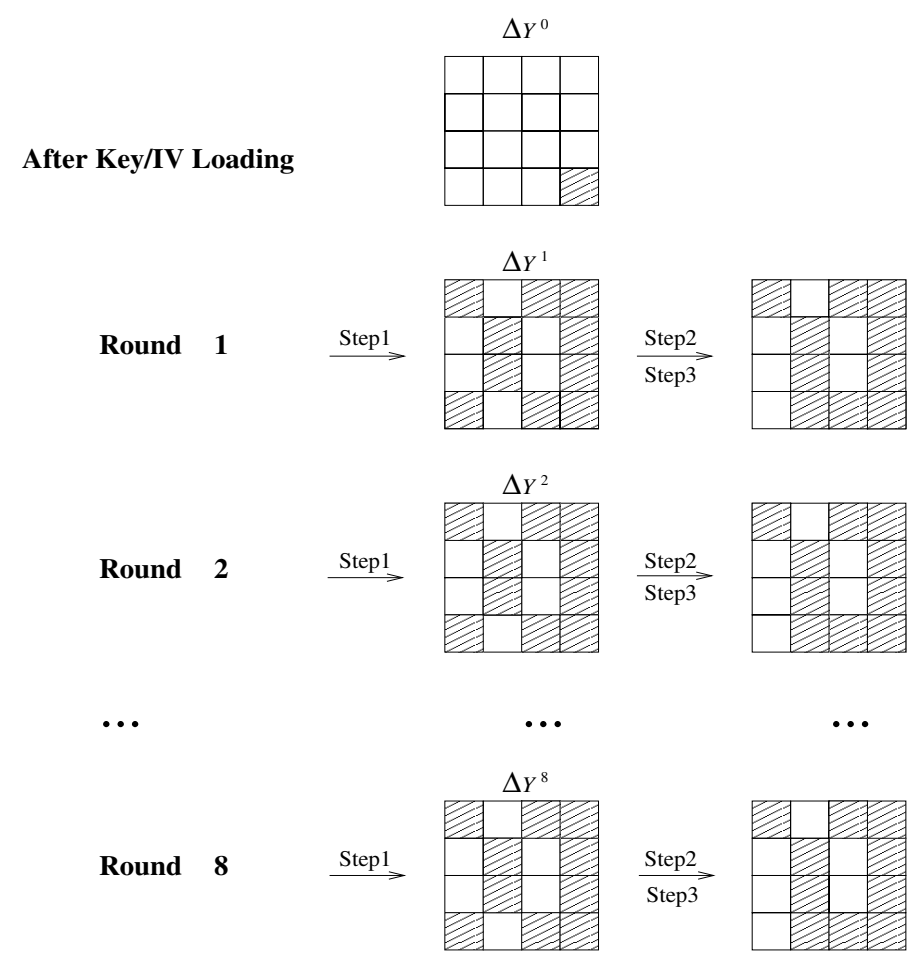

Fig. 2. The differential characteristic $\Omega_{Y}$ in the state initialization

It is obvious that, if $I V$ and $I V^{\prime}$ with the same key $K$ are different at only one bit $\Delta i v_{66}=1$, the initial state differential $\Delta X^{0}$ is $(0,0,0,0)^{T}$ and $\Delta Y^{0}$ is $(0,0,0,4)^{T}$. And then, by the equations (2) and (3), it is easy to confirm that, $\Delta X^{0}$ and the choice of $\Delta Y^{1}$ guarantee the keystream output differential $\Delta z_{r}^{1}$ at the first round is 0 . So, from Step 3 of the warm-up process in Subsection 2.2, the difference $\Delta X^{1}$ is always $(0,0,0,0)^{T}$ after the first round. Similarly, $\Delta X^{1}$ and the choice of $\Delta Y^{2}$ guarantee $\Delta X^{2}$ is always $(0,0,0,0)^{T}$ after the second round, and so on. This means that, under the condition $I V$ and $I V^{\prime}$ are different at only one bit $\Delta i v_{66}=1$, if the differential characteristic $\Omega_{Y}$ holds, then the 
differential characteristic $\Omega_{X}$ holds with probability 1 . And we can only focus on the occurrence probability of $\Omega_{Y}$ in the next sections.

\section{Chosen IV Differential Attack on TSC-4}

In this section, we introduce a chosen IV differential attack on TSC-4.

\subsection{Weak Keys Resulting in the High Occurrence Probability of Differential Characteristic $\Omega_{Y}$}

We now investigate what keys and IVs can result in the high occurrence probability of differential characteristic $\Omega_{Y}$ in Section 3 .

Experiment 1. For each $j_{0}\left(j_{0}=64, \cdots, 79\right)$, we randomly select $2^{38}(K, I V$, $\left.I V^{\prime}\right)$ triplets where $I V$ and $I V^{\prime}$ are only different at one bit $\Delta i v_{j_{0}}=1$. For each $\left(K, I V, I V^{\prime}\right)$ triplet, we perform two state initialization processes with $(K, I V)$ and $\left(K, I V^{\prime}\right)$ to search for $\Omega_{Y}$. We find that there are more $\Omega_{Y}$ holding for $j_{0}=66$ or 68 . Especially, for $j_{0}=66$, the number of obtained $\Omega_{Y}$ is up to 4983 . Since each $\Omega_{Y}$ corresponds to one $(K, I V)$ pair, we can further investigate the statistical property of $4983(K, I V)$ pairs obtained above. Firstly, we perform one Key/IV loading process with each obtained $(K, I V)$ pair, then 4983 initial state $Y^{0}$ can be obtained. Secondly, we employ a $\chi^{2}$ test for 16 states of each $\left[Y^{0}\right]_{i}(i=0,1, \cdots, 31)$. Finally, list the $\chi^{2}$ distributions of $\left[Y^{0}\right]_{i}$ which are serious unbalance, and the result is illustrated in Table 1.

Table 1. $\chi^{2}$ distributions and $\chi^{2}$ values of $\left[Y^{0}\right]_{i}(i=0,1, \cdots, 4)$

\begin{tabular}{|c|c|c|c|c|c|}
\hline$k$ & {$\left[Y^{0}\right]_{0}$} & {$\left[Y^{0}\right]_{1}$} & {$\left[Y^{0}\right]_{2}$} & {$\left[Y^{0}\right]_{3}$} & {$\left[Y^{0}\right]_{4}$} \\
\hline \hline 0 & 169 & 335 & 0 & 636 & 58 \\
1 & 156 & 50 & 0 & 73 & 0 \\
2 & 229 & 57 & 4 & 63 & 98 \\
3 & 379 & 103 & 5 & 624 & 0 \\
4 & 183 & 71 & 0 & 97 & $\mathbf{4 5 9 4}$ \\
5 & $\mathbf{3 7 6}$ & 101 & 0 & $\mathbf{5 6 3}$ & 15 \\
6 & $\mathbf{4 0 0}$ & 116 & 0 & $\mathbf{6 0 2}$ & 1 \\
7 & 356 & 839 & 0 & 50 & 7 \\
8 & 176 & 63 & 0 & 93 & 30 \\
9 & $\mathbf{3 5 9}$ & 115 & 0 & $\mathbf{7 0 7}$ & 1 \\
10 & $\mathbf{4 0 6}$ & 92 & 0 & $\mathbf{6 6 5}$ & 4 \\
11 & 323 & 841 & 0 & 47 & 4 \\
12 & 322 & 105 & 12 & 596 & 1 \\
13 & 318 & $\mathbf{8 6 5}$ & 9 & 68 & 76 \\
14 & 333 & $\mathbf{7 9 1}$ & $\mathbf{2 4 1 3}$ & 64 & 1 \\
15 & 498 & 439 & $\mathbf{2 5 4 0}$ & 35 & 93 \\
\hline \hline$\chi^{2}$ & 486.2 & 5201.4 & 34429.2 & 4047.9 & 62874.7 \\
\hline
\end{tabular}


From Table 1, we find that the 5 least significant columns of $\left[Y^{0}\right]_{i}$ reveal the serious unbalance for $\chi^{2}$ value which is more than 100 , where $\chi^{2}=\sum_{k=0}^{15} \frac{\left(n_{k}-n p_{k}\right)^{2}}{n p_{k}}$, $n_{k}$ is the frequency of the 4-bit integer $\left[Y^{0}\right]_{i}$ being $k, n=\sum_{k=0}^{15} n_{k}$ and $p_{k}=\frac{1}{16}$. Since $K$ and $I V$ are embedded into $Y^{0}$, we hope to deduce some linear correlations between $K$ and $I V$ by the unbalance of $\left[Y^{0}\right]_{i}$.

For the initial state $Y^{0}=\left(y_{3}^{0}, y_{2}^{0}, y_{1}^{0}, y_{0}^{0}\right)^{T}$ initialed by $I V$ and $K$ after the Key/IV loading process, we know that $\left[y_{0}^{0}\right]_{i}=i v_{64+i},\left[y_{1}^{0}\right]_{i}=i v_{16+i},\left[y_{2}^{0}\right]_{i}=k_{64+i}$ and $\left[y_{3}^{0}\right]_{i}=k_{16+i}$, where $i=0,1, \cdots, 4$. So, $\left[Y^{0}\right]_{i}$ can be represented as

$$
\left[Y^{0}\right]_{i}=i v_{64+i}+i v_{16+i} \cdot 2+k_{64+i} \cdot 2^{2}+k_{16+i} \cdot 2^{3}, i=0,1, \cdots, 4 .
$$

Especially, we can find some strong linear correlations between $K$ and $I V$. For example, $\left[Y^{0}\right]_{4}$ concentrates on the point $0 \times 4$ with probability $\frac{4594}{4983}=0.9129$ by the sixth column of Table 1 . So from $\left[Y^{0}\right]_{4}=0 \times 4$ and equation (4), we get the following conditional probability

$$
\operatorname{Pr}\left(k_{20}=0, k_{68}=1, i v_{20}=0, i v_{68}=0 \mid \Omega_{Y}\right)=0.9129 .
$$

We try to explore some other more complex linear correlations between $K$ and $I V$ by observing the $\chi^{2}$ distribution of $\left[Y^{0}\right]_{i}$. We firstly investigate the possible linear equations from $\left[Y^{0}\right]_{0}$. In order to find the linear correlations easily with

\begin{tabular}{|c|c|c|c|c|}
\hline & 00 & 01 & 10 & 11 \\
\hline 00 & 169 & 156 & 229 & 379 \\
\hline 01 & 183 & 376 & 400 & 356 \\
\hline 10 & 176 & 359 & 406 & 323 \\
\hline 1 & 322 & 318 & 333 & 498 \\
\hline
\end{tabular}
high probability, we list the distribution of $\left[Y^{0}\right]_{0}$ as a matrix in Fig. 3.

Fig. 3. $4 \times 4$ matrix of $[Y]_{0}, a b$ represents $k_{16} k_{64}$, and $c d$ is $i v_{16} i v_{64}$

From Fig. 3, we know that two linear equations $i v_{64} \oplus i v_{16}=1$ and $k_{64} \oplus k_{16}=1$ hold concurrently in the four bold items which correspond to $5=(0101)_{2}, 6=$ $(0110)_{2}, 9=(1001)_{2}$ and $10=(1010)_{2}$ respectively.

From $\operatorname{Pr}\left([Y]_{0}=5,6,9,10 \mid \Omega_{Y}\right)=\frac{1541}{4983}=0.3093$, we get

$$
\operatorname{Pr}\left(i v_{64} \oplus i v_{16}=1, k_{64} \oplus k_{16}=1 \mid \Omega_{Y}\right)=0.3093 .
$$

Clearly, the probability of (6) is much more than the average value 0.25 . 
By studying the 3rd, 4-th and 5-th columns of Table 1, we can conclude that

$$
\begin{aligned}
& \operatorname{Pr}\left(k_{17}=1, k_{65}=1, i v_{65} \oplus i v_{17}=1 \mid \Omega_{Y}\right)=0.3323, \\
& \operatorname{Pr}\left(k_{18}=1, k_{66}=1, i v_{18}=1 \mid \Omega_{Y}\right)=0.9939 \\
& \operatorname{Pr}\left(k_{19} \oplus k_{67}=1, i v_{19} \oplus i v_{67}=1 \mid \Omega_{Y}\right)=0.5091 .
\end{aligned}
$$

Summing up all the linear equations (5)-(9), we define the following sets,

$$
\begin{aligned}
& A_{0}=\left\{K \mid k_{64} \oplus k_{16}=1\right\}, \\
& A_{1}=\left\{K \mid k_{17}=1, k_{65}=1\right\}, \\
& A_{2}=\left\{K \mid k_{18}=1, k_{66}=1\right\}, \\
& A_{3}=\left\{K \mid k_{19} \oplus k_{67}=1\right\}, \\
& A_{4}=\left\{K \mid k_{20}=0, k_{68}=1\right\} . \\
& B_{0}=\left\{I V \mid i v_{64} \oplus i v_{16}=1\right\}, \\
& B_{1}=\left\{I V \mid i v_{65} \oplus i v_{17}=1\right\}, \\
& B_{2}=\left\{I V \mid i v_{18}=1\right\}, \\
& B_{3}=\left\{I V \mid i v_{19} \oplus i v_{67}=1\right\}, \\
& B_{4}=\left\{I V \mid i v_{20}=0, i v_{68}=0\right\} .
\end{aligned}
$$

Let

$$
\mathscr{A}=\bigcap_{j=0}^{4} A_{j}, \quad \mathscr{B}=\bigcap_{j=0}^{4} B_{j} .
$$

We can get the following probability by searching $4983(K, I V)$ pairs in Experiment 1.

$$
\operatorname{Pr}\left(K \in \mathscr{A}, I V \in \mathscr{B} \mid \Omega_{Y}\right)=\frac{386}{4983}=2^{-3.69} .
$$

And it is obvious that

$$
\operatorname{Pr}\left(\Omega_{Y}\right)=\frac{4983}{2^{38}}=2^{-25.71} .
$$

Because $\operatorname{Pr}(K \in \mathscr{A}, I V \in \mathscr{B})=2^{-14}$, by the conditional probability formula with (10) and (11), we get

$$
\operatorname{Pr}\left(\Omega_{Y} \mid K \in \mathscr{A}, I V \in \mathscr{B}\right)=\frac{\operatorname{Pr}\left(K \in \mathscr{A}, I V \in \mathscr{B} \mid \Omega_{Y}\right) \operatorname{Pr}\left(\Omega_{Y}\right)}{\operatorname{Pr}(K \in \mathscr{A}, I V \in \mathscr{B})}=2^{-15.40} .
$$

We define the key $K$ which falls into set $\mathscr{A}$ as a weak key. For 80 bits key size, the number of weak keys is $2^{80-8}=2^{72}$. From equation (12), we know that, for a chosen IV pair $\left(I V, I V^{\prime}\right)$ with $\left\{I V, I V^{\prime}\right\} \subset \mathscr{B}$, if $K$ is a weak key, $\Omega_{Y}$ occurs with high probability $2^{-15.40}$. We can verify the above probability by the statistical Experiment 2. 
Experiment 2. We randomly select $2^{10}$ weak keys. For each weak key, randomly select $2^{20}\left(I V, I V^{\prime}\right)$ pairs where $I V$ and $I V^{\prime}$ are only different at one bit $\Delta i v_{66}=$ 1 , and both belong to set $\mathscr{B}$. Then we get the total occurrence frequency of the differential characteristic $\Omega_{Y}$ is 26086 .

Experiment 2 means that $\operatorname{Pr}\left(\Omega_{Y} \mid K \in \mathscr{A}, I V \in \mathscr{B}\right)$ is about $\frac{26086}{2^{10} \times 2^{20}}=2^{-15.33}$ which confirms our result in (12) again.

\subsection{Identifying and Recovering the Weak Keys}

From the above subsection, we know that, for a chosen IV pair, if $K$ is a weak key, $\Omega_{Y}$ happens with high probability $2^{-15.40}$. But this is not enough to identify and recover a weak key, we need more information. Denote the first actual keystream output byte which is used to encrypt a plaintext byte as $z_{s}^{1}$, and $z_{s}^{1}$ is generated by $I V$ and $K$. Similarly, $z_{s}^{\prime 1}$ corresponds to $I V^{\prime}$ and $K$. Let $\Delta z_{s}^{1}$ be $z_{s}^{1} \oplus z_{s}^{\prime 1}$, and $\left[\Delta z_{s}^{1}\right]_{k}$ be the $k$-th least significant bit of the differential byte $\Delta z_{s}^{1}, k=0,1, \cdots 7$. Then the bias of $\left[\Delta z_{s}^{1}\right]_{k}$ will provide a solution to identify a weak key.

Experiment 3. In Experiment 2, we have obtained 26086 differential characteristics $\Omega_{Y}$. And we know each $\Omega_{Y}$ corresponds to one $\left(K, I V, I V^{\prime}\right)$ triplet, then we utilize each $\left(K, I V, I V^{\prime}\right)$ triplet to run cipher body once and produce two first 8-bit keystream outputs $z_{s}^{1}$ and $z_{s}^{\prime 1}$. Then $26086 \Delta z_{s}^{1}$ bytes can be obtained.

Denote the number of $\left[\Delta z_{s}^{1}\right]_{k}$ being ' 0 ' as $n_{k, 0}$, and the number of $\left[\Delta z_{s}^{1}\right]_{k}$ being ' 1 ' as $n_{k, 1}$. The $0-1$ bias of $\left[\Delta z_{s}^{1}\right]_{k}$ can be represented as $\varepsilon_{k}=\left|\frac{n_{k, 0}}{n_{k, 0}+n_{k, 1}}-\frac{1}{2}\right|$. From Experiment 3, for a weak key with chosen IV pairs, if the differential characteristic $\Omega_{Y}$ occurs, the bias of $\left[\Delta z_{s}^{1}\right]_{k}$ is listed in Table 2.

Table 2. The bias of $\left[\Delta z_{s}^{1}\right]_{k}$ with weak keys $(k=0,1, . ., 7)$

\begin{tabular}{|c|c|c|c|c|c|c|c|c|}
\hline$k$ & 0 & 1 & 2 & 3 & 4 & 5 & 6 & 7 \\
\hline$\left|\log _{2} \varepsilon_{k}\right|$ & 4.38 & $\mathbf{3 . 5 4}$ & 5.09 & 5.90 & 4.91 & 4.81 & 6.15 & 5.02 \\
\hline
\end{tabular}

For a weak key with chosen IV pairs, we adopt $\left[\Delta z_{s}^{1}\right]_{1}$ which holds with the highest bias, and get the following conclusion by Experiment 3 .

$$
\operatorname{Pr}\left(\left[\Delta z_{s}^{1}\right]_{1}=0 \mid \Omega_{Y}, K \in \mathscr{A}, I V \in \mathscr{B}\right)=\frac{1}{2}+2^{-3.54} .
$$

In an actual attack, we can not get $\Omega_{Y}$ directly except for the actual keystream output. Then we need to know the bias of $\left[\Delta z_{s}^{1}\right]_{1}$ without the condition $\Omega_{Y}$. By further experiments, we can find that if $\Omega_{Y}$ does not happen, for any $K$ and $I V$, the probability $\operatorname{Pr}\left(\left[\Delta z_{s}^{1}\right]_{1}=0 \mid \bar{\Omega}_{Y}\right)$ is very close to $\frac{1}{2}$. Then for a weak key and the chosen IV pairs, from (12) and (13), we can get

$$
\operatorname{Pr}\left(\left[\Delta z_{s}^{1}\right]_{1}=0 \mid K \in \mathscr{A}, I V \in \mathscr{B}\right)=\frac{1}{2}+2^{-15.40} \times 2^{-3.54}=\frac{1}{2}+2^{-18.94} .
$$


In order to improve the correctness for identifying a weak key, we need to explore the $0-1$ bias of $\left[\Delta z_{s}^{1}\right]_{k}$ for a strong key. A strong key means that the key $K \in \overline{\mathscr{A}}$.

Utilizing 4983 keys and IV pairs obtained by Experiment 1, we get the probability

$$
\operatorname{Pr}\left(K \in \overline{\mathscr{A}}, I V \in \mathscr{B} \mid \Omega_{Y}\right)=\frac{151}{4983}=2^{-5.04} .
$$

Since $\operatorname{Pr}(K \in \overline{\mathscr{A}}, I V \in \mathscr{B})=\left(1-2^{-8}\right) \times 2^{-6}=2^{-6.01}$, from (15), it is easy to deduce the probability

$$
\operatorname{Pr}\left(\Omega_{Y} \mid K \in \overline{\mathscr{A}}, I V \in \mathscr{B}\right)=\frac{\operatorname{Pr}\left(K \in \overline{\mathscr{A}}, I V \in \mathscr{B} \mid \Omega_{Y}\right) \operatorname{Pr}\left(\Omega_{Y}\right)}{\operatorname{Pr}(K \in \overline{\mathscr{A}}, I V \in \mathscr{B})}=2^{-24.74} .
$$

Experiment 4. We randomly select $2^{10}$ strong keys. For each strong key, randomly select $2^{24}$ chosen IV pairs, and we can get that the total frequency that $\Omega_{Y}$ occurs is 645 . Therefore $\operatorname{Pr}\left(\Omega_{Y} \mid K \in \overline{\mathscr{A}}, I V \in \mathscr{B}\right)$ is about $\frac{645}{2^{10} \times 2^{24}}=2^{-24.67}$. It is clear that, Experiment 4 verifies the result in (16).

In Experiment 4, we can also get $645 \Delta z_{s}^{1}$ corresponding to $645 \Omega_{Y}$. By counting the number of ' 0 ' among $645\left[\Delta z_{s}^{1}\right]_{1}$, we calculate the following probability under a strong key and the chosen IV pairs,

$$
\operatorname{Pr}\left(\left[\Delta z_{s}^{1}\right]_{1}=0 \mid \Omega_{Y}, K \in \overline{\mathscr{A}}, I V \in \mathscr{B}\right)=\frac{1}{2}+2^{-3.68} .
$$

By combination of equations (16) and (17), for a strong key and the chosen IV pairs, similarly to (14), we get the probability

$$
\operatorname{Pr}\left(\left[\Delta z_{s}^{1}\right]_{1}=0 \mid K \in \overline{\mathscr{A}}, I V \in \mathscr{B}\right)=\frac{1}{2}+2^{-24.74} \times 2^{-3.68}=\frac{1}{2}+2^{-28.42} .
$$

We use (14) and (18) to identify the weak keys. Approximate the binomial distribution with the normal distribution. Denote the total number of samples as $N$, the mean as $\mu$, and the standard variance as $\sigma$.

From (14), $p=\frac{1}{2}+2^{-18.94}, \mu=N p$ and $\sigma=\sqrt{N p(1-p)}$.

From (18), $p^{\prime}=\frac{1}{2}+2^{-28.42}, \mu^{\prime}=N p^{\prime}$ and $\sigma^{\prime}=\sqrt{N p^{\prime}\left(1-p^{\prime}\right)}$.

For the normal distribution, the cumulative function gives value $1-2^{-9.53}$ at $3 \sigma^{\prime}$, and value 0.023 at $-2 \sigma$.

If the following relation holds

$$
\mu-\mu^{\prime} \geqslant 3 \sigma^{\prime}+2 \sigma,
$$

a strong key will be wrongly identified as a weak key (false positive) with probability $2^{-9.53}$, and each weak key is not identified as a weak key (false negative) with probability 0.023 . This means that the weak keys can be successfully identified. By solving (19), the amount of IV pairs required is $N=2^{40.53}$.

Identifying a weak key means that we can recover 8 key bits. The other 72 key bits can be recovered by searching exhaustively. 


\section{Conclusion}

In this paper, we developed a differential attack on TSC-4. Utilizing the structure of T-function in TSC-4, we constructed a special differential characteristic with high probability which incurs about $2^{72}$ weak keys. $2^{40.53}$ chosen IV pairs can be used to identify a weak key and recover 8 bits of the weak key. We can recover the other 72 key bits by exhaustive search.

\section{References}

1. Hong, J., Lee, D.H., Yeom, Y., Han, D.: New Class of Single Cycle T-functions. In: Gilbert, H., Handschuh, H. (eds.) FSE 2005. LNCS, vol. 3557, pp. 68-82. Springer, Heidelberg (2005)

2. Hong, J., Lee, D.H., Yeom, Y., Han, D., Chee, S.: T-function Based Stream Cipher TSC-3, available at http://www.ecrypt.eu.org/stream/ciphers/tsc3/tsc3.pdf

3. Fischer, S., Meier, W., Berbain, C., et al.: Non-randomness is eSTREAM Candidates Salsa20 and TSC-4. In: Barua, R., Lange, T. (eds.) INDOCRYPT 2006. LNCS, vol. 4329, pp. 2-16. Springer, Heidelberg (2006)

4. Klimov, A., Shamir, A.: A New Class of Invertible Mappings. In: Kaliski Jr., B.S., Koç, Ç.K., Paar, C. (eds.) CHES 2002. LNCS, vol. 2523, pp. 470-483. Springer, Heidelberg (2003)

5. Klimov, A., Shamir, A.: Cryptographic Application of T-functions. In: Matsui, M., Zuccherato, R.J. (eds.) SAC 2003. LNCS, vol. 3006, pp. 248-261. Springer, Heidelberg (2004)

6. Klimov, A., Shamir, A.: New Cryptographic Primitives Based on Multiword Tfunctions. In: Roy, B., Meier, W. (eds.) FSE 2004. LNCS, vol. 3017, pp. 1-15. Springer, Heidelberg (2004)

7. Kunzli, S., Junod, P., Meier, W.: Distinguishing Attacks on T-functions. In: International Conference on Cryptology in Malaysia (2005)

8. Mitra, J., Sarkar, P.: Time-memory Trade-Off Attacks on Multiplications and Tfunctions. In: Lee, P.J. (ed.) ASIACRYPT 2004. LNCS, vol. 3329, pp. 468-482. Springer, Heidelberg (2004)

9. Moon, D., Kwon, D., Han, D., et al.: T-function Based Stream Cipher TSC-4, available at http://www.ecrypt.eu.org/stream/p2ciphers/tsc4/tsc4_p2.pdf

10. Muller, F., Peyrin, T.: Linear Cryptanalysis of the TSC Family of Stream Ciphers. In: Roy, B. (ed.) ASIACRYPT 2005. LNCS, vol. 3788, pp. 373-394. Springer, Heidelberg (2005)

11. Muller, F., Peyrin, T.: Linear Cryptanalysis of TSC Stream Ciphers - Applications to the ECRYPT Proposal TSC-3, available at http://www.ecrypt.eu.org/stream/papersdir/042.ps 